\title{
Feasibility of reirradiation and treatment outcome in a previously irradiated territory in head-and-neck malignancies Sweety Gupta ${ }^{\mathrm{a}}$, Amit Gupta ${ }^{\mathrm{b}}$, Prekshi Choudhary ${ }^{\mathrm{c}}$, Ashish Gupta ${ }^{\mathrm{b}}$, Manjari Shah ${ }^{d}$, Shashank Srinivasan ${ }^{\dagger}$, Ruchir Tandon ${ }^{\mathrm{e}}$, Sudarsan De ${ }^{\mathrm{d}}$
}

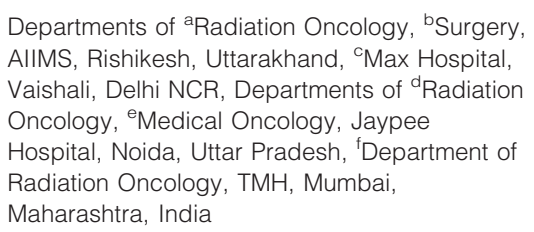

Departments of a Radiation Oncology, ${ }^{\mathrm{b}}$ Surgery, AlIMS, Rishikesh, Uttarakhand, 'Max Hospital, Vaishali, Delhi NCR, Departments of ${ }^{\mathrm{d}}$ Radiation Oncology, ${ }^{e}$ Medical Oncology, Jaypee

Hospital, Noida, Uttar Pradesh, 'Department of Radiation Oncology, TMH, Mumbai,

Maharashtra, India

Correspondence to Sweety Gupta, MBBS, MD, Department of Radiation Oncology, 5th floor, Medical College Block, AllMS, Rishikesh,

Uttarakhand, 249203, India. Tel: +989198 1332;

e-mail: drsg2411@yahoo.co.in

Received 6 June 2018

Accepted 6 September 2018

The Egyptian Journal of Otolaryngology 2018, 34:283-288

\begin{abstract}
Introduction
Despite combined modality treatment, local persistent or recurrent disease is a primary cause of treatment failure in $30-50 \%$ of patients with advanced head-andneck cancer (HNC). The role of reirradiation is feared because of increased risk of toxicity due to the previously irradiated target volume. In the present study, we have evaluated reirradiation by image-guided intensity-modulated radiotherapy in recurrent and second primary HNCs and its outcome.
\end{abstract}

Materials and methods

In all, 24 patients with recurrence or second primary tumors in HNC were included in the study conducted between January 2009 to August 2014. All patients were treated by the image-guided intensity-modulated radiotherapy technique. The most common site of recurrence/second primary was oral tongue. The time interval between initial radiotherapy and reirradiation ranged from 8 months to 17 years. Statistical analysis used

The association between qualitative variables was tested using the $\chi^{2}$-test. Statistical significance was interpreted using an arbitrary cut-off of $P=0.05$. Kaplan-Meier Survival graph was plotted to depict the survival pattern of the study patients.

\section{Results}

The planning target volume volume of reirradiation ranged from 26.72 to $469.32 \mathrm{ml}$ (median: $118.71 \mathrm{ml}$ ). Toxicity was more in patients receiving concurrent chemoradiation and in patients with less interval time between reirradiation. Out of the 24 patients, nine were no evidence of disease, eight patients expired (five disease progression, two comorbidities, and one due to hematemesis after the development of third primary), two recurred, one developed distant metastases, and four were lost to follow-up.

Conclusion

Surgical resection, with or without reirradiaton, provides the highest likelihood for successful salvage in locoregional (LR) recurrences in HNCs. Patients reirradiated after a long time from initial irradiation have better tolerance.

\section{Keywords:}

image-guided intensity-modulated radiotherapy, reirradiation, second malignancy, surgery

Egypt J Otolaryngol 34:283-288

(C) 2018 The Egyptian Journal of Otolaryngology

$1012-5574$

\section{Introduction}

Incidence of recurrence in head-and-neck cancers (HNCs) is in the order of $10-15 \%$ for early stage disease, $15-25 \%$ for intermediate stage disease, and $30-50 \%$ for advanced stage disease [1]. For those who did not develop locoregional (LR) or distant recurrence, there is still a risk of $25 \%$ at 5 years to develop a second primary HNC [2]. Both recurrent tumors and second malignancies are difficult to manage when they occur in a previously irradiated area and are considered a challenging problem for the whole team. For decades, the management of recurrent and second primary $\mathrm{HNC}$ in the previously irradiated territory has been limited to salvage surgery in selected patients resulting in a 2-year disease-free survival of $36.3 \%$ (range: $0-59 \%$ ) and a 5-year overall survival of 36\% (range: 23-55\%) [3,4]. The role of external reirradiation was rarely investigated mainly due to fear of severe toxicity from high doses to large, previously irradiated target volumes.

Intensity-modulated radiotherapy permits extremely conformal delivery of definitive doses with sparing of neighboring normal tissues, so promises to improve both efficacy and morbidity in reirradiated patients [5].

This is an open access journal, and articles are distributed under the terms of the Creative Commons Attribution-NonCommercial-ShareAlike 4.0 License, which allows others to remix, tweak, and build upon the work non-commercially, as long as appropriate credit is given and the new creations are licensed under the identical terms. 


\section{Materials and methods}

Between January 2009 and August 2014, 24 patients who underwent reirradiation at our institute for recurrence and second primary $\mathrm{HNC}$ were included in this retrospective study (Table 1). IEC approval was taken to conduct study. The patients received external beam reirradiation to the head-and-neck area with curative intent to overlapping areas that had previously been treated with radiotherapy.

The following selection criteria were applied: histologic proof of recurrent disease or second primary tumor after previous radiation and no evidence of distant metastases. The diagnostic evaluation included physical examination, radiologic evaluation of the head and neck by computed tomography (CT), and/ or magnetic resonance imaging, screening for distant metastases using CT and/or positron emission tomography $(\mathrm{CT})$. For previously irradiated patients presenting with recurrent or second primary tumors, surgical salvage has remained the standard of care in our clinical practice. In cases of unresectability, primary reirradiation (with or without concurrent chemotherapy) was considered as a therapeutic option. Warren and Gate's criteria [6-8] were used to classify tumors as second primary or recurrence which included: (a) histologic confirmation of malignancy in both the index and secondary tumors; (b) the two tumors should be separated by normal mucosa of at least $2 \mathrm{~cm}$ and if they are in the same anatomic location, then there must be a gap of at least 5 years between their occurrence; and (c) the possibility

Table 1 General characteristics of the study population $(\mathrm{n}=\mathbf{2 4 )}$

\begin{tabular}{lc}
\hline Characteristics & $n(\%)$ \\
\hline Sex & \\
Male & $19(79.2)$ \\
Female & $5(20.8)$ \\
Surgery & \\
$\quad$ No & $14(58.3)$ \\
Yes & $10(41.7)$ \\
Concurrent chemotherapy & \\
No & $18(75.0)$ \\
Yes & $6(25.0)$ \\
Toxicity (grade) & \\
1 & $6(25.0)$ \\
2 & $7(29.2)$ \\
3 & $11(45.8)$ \\
Outcome & \\
No evidence of disease & $9(37.5)$ \\
Expired & $8(33.4)$ \\
Recurrence & $2(8.3)$ \\
Metastasis & $1(4.2)$ \\
Lost to follow-up & $4(16.7)$ \\
\hline
\end{tabular}

of the second malignancy being metastasis of the index tumor must be ruled out. Postoperative reirradiation was considered only if the pathologic features of the surgical specimen indicated a high risk of subsequent recurrence: tumor size, positive margins, lymph node metastasis with extracapsular extension, and/or multiple lymph node metastases.

\section{Treatment}

Patients were immobilized with a thermoplastic headand-neck mask including the shoulders, to ensure reproducibility of reirradiation. CT simulation with a 2-mm slice thickness was performed in all patients. Targets and organs at risk were delineated on axial CT scan sections according to the RTOG contouring guidelines. Gross tumor volume encompassing tumor and/or pathological lymph nodes was expanded to form the clinical target volume (CTV). In case of surgery, the CTV encompassed the postoperative tumor bed. The CTV was expanded isotropically with a $5 \mathrm{~mm}$ margin to a planning target volume (PTV). No prophylactic neck irradiation was done.

\section{Dose prescription}

Treatment planning was done on the ECLIPSE treatment planning system. Inverse planning intensitymodulated radiotherapy (IMRT) was carried out for all the patients followed by evaluation of target coverage, dose uniformity, and dose to normal structures. Dose prescription aimed to deliver at least $95 \%$ of the prescribed dose to at least $95 \%$ of the PTV and not more than $107 \%$ of the prescribed dose to not more than $5 \%$ of the PTV (Fig. 1).

The treatment was delivered using 6-MV photons by a linear accelerator (CLINAC iX, @ Varian system,CA). Setup verification was done using Cone Beam CT/2D kV imaging. A weekly offline review was performed (Fig. 2).

The patients were seen at least once a week by a radiation oncologist who assessed acute toxicity according to the Common Toxicity Criteria 3.0. Weekly blood investigations were performed which included a complete blood count and evaluation of renal parameters for patients receiving concurrent chemotherapy. Patients were subsequently followed up every 1-2 months for the first year, 3 months for the second years, and 4-6 months in subsequent years. Response evaluation was done by MRI neck or PET CT scan 3 months after treatment.

\section{Statistical methods}

Data were entered into MS Excel and analyzed using SPSS v20. Qualitative data were summarized as 


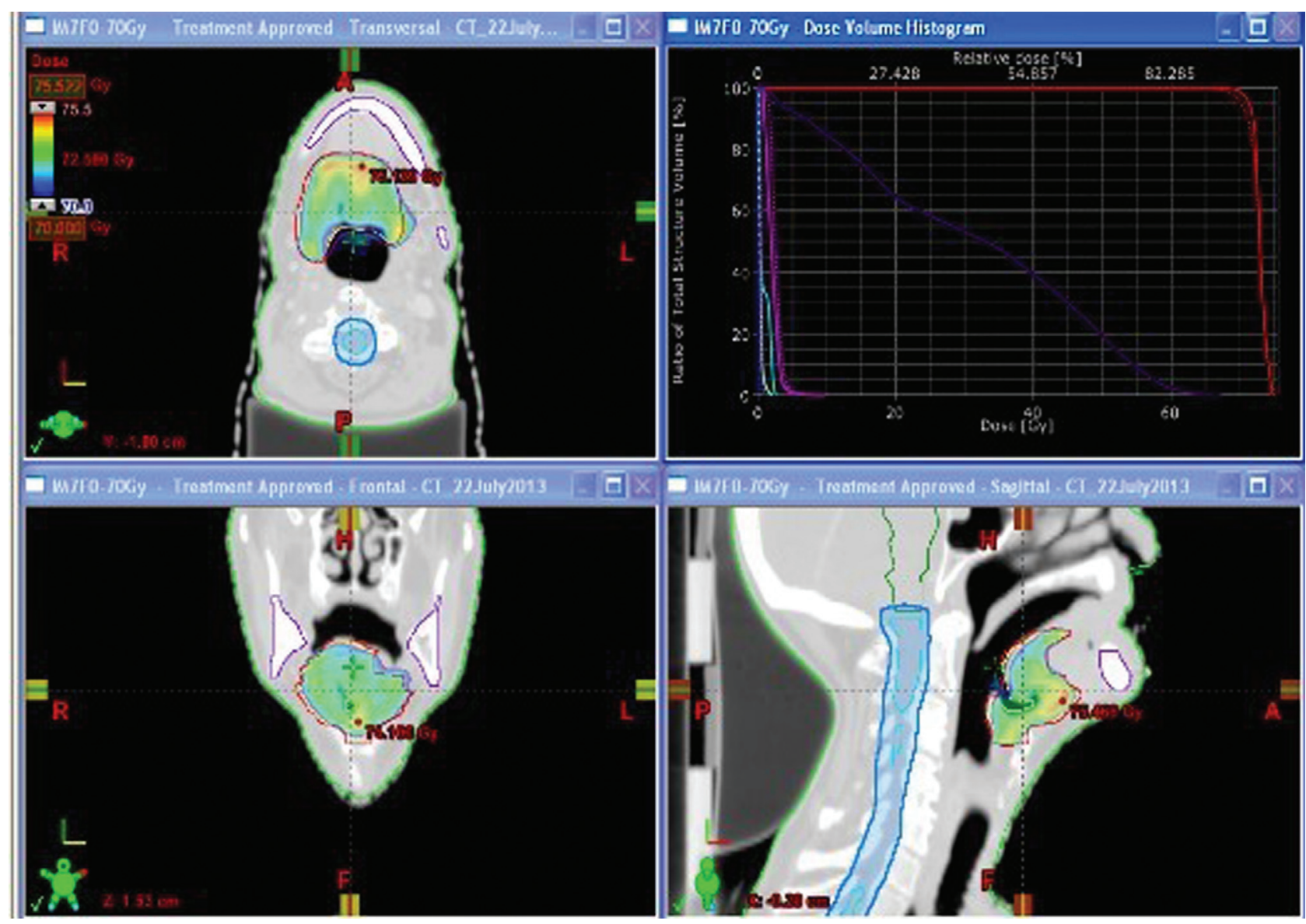

Inverse planning intensity-modulated radiotherapy plan.

frequencies and percentages. Quantitative data were checked for normality. Normally distributed data were summarized using mean and SD. Non-normally distributed data were summarized using median and interquartile range. The association between qualitative variables was tested using the $\chi^{2}$-test. Kruskal-Wallis test was used to look for association between toxicity and time to recurrence and PTV volume. Statistical significance was interpreted using an arbitrary cut-off of $P=0.05$. Kaplan-Meier survival graph was plotted to depict the survival pattern of the study patients and the median survival was calculated.

\section{Results}

The mean patient age was 56 years (range: $38-82$ years) and $79 \%$ of the patients were men; $13(54 \%)$ had second primary and $11(46 \%)$ had recurrence. Of the 24 patients, 11 had recurrence in the oral cavity, four in the oropharynx, two each in the hypopharynx and larynx, one in the nasopharynx, and two had contralateral nodal recurrence. The time interval between initial radiotherapy and reirradiation ranged from 8 months to 17 years. Salvage surgery preceded radiotherapy in 10 (42\%) patients and $14(58 \%)$ underwent radical radiotherapy; nine (37.5\%) patients received concurrent chemotherapy with radiation therapy. PTV included CTV with a margin in postoperative patients and gross tumor with margin in radical patients. No prophylactic nodal irradiation was done. The PTV volume of reirradiation ranged from 26.72 to $469.32 \mathrm{ml}$ (median: $118.71 \mathrm{ml}$ ). The PTV dose ranged from 45 to $70.3 \mathrm{~Gy}$ with a median dose of $60 \mathrm{~Gy}$. Dmax to the spine ranged from 2.08 to $23 \mathrm{~Gy}$ (mean dose: $5.09 \mathrm{~Gy}$ ). Dmax to the brainstem ranged from 0.36 to $37.4 \mathrm{~Gy}$ (mean dose: $4.83 \mathrm{~Gy}$ ). The most common acute side effect was mucositis. Grade 3 mucositis was seen in 11 patients. Toxicity was more in patients receiving concurrent chemoradiation, patients with lesser time interval between primary irradiation and reirradiation and with greater PTV volumes. At last follow-up, nine (37.5\%) patients have no evidence of disease, eight (33.3\%) patients expired (five due to disease progression, two due to comorbidities, and one due to hematemesis after the development of third primary), two (8.3\%) recurred, one (4.1\%) developed 
Figure 2

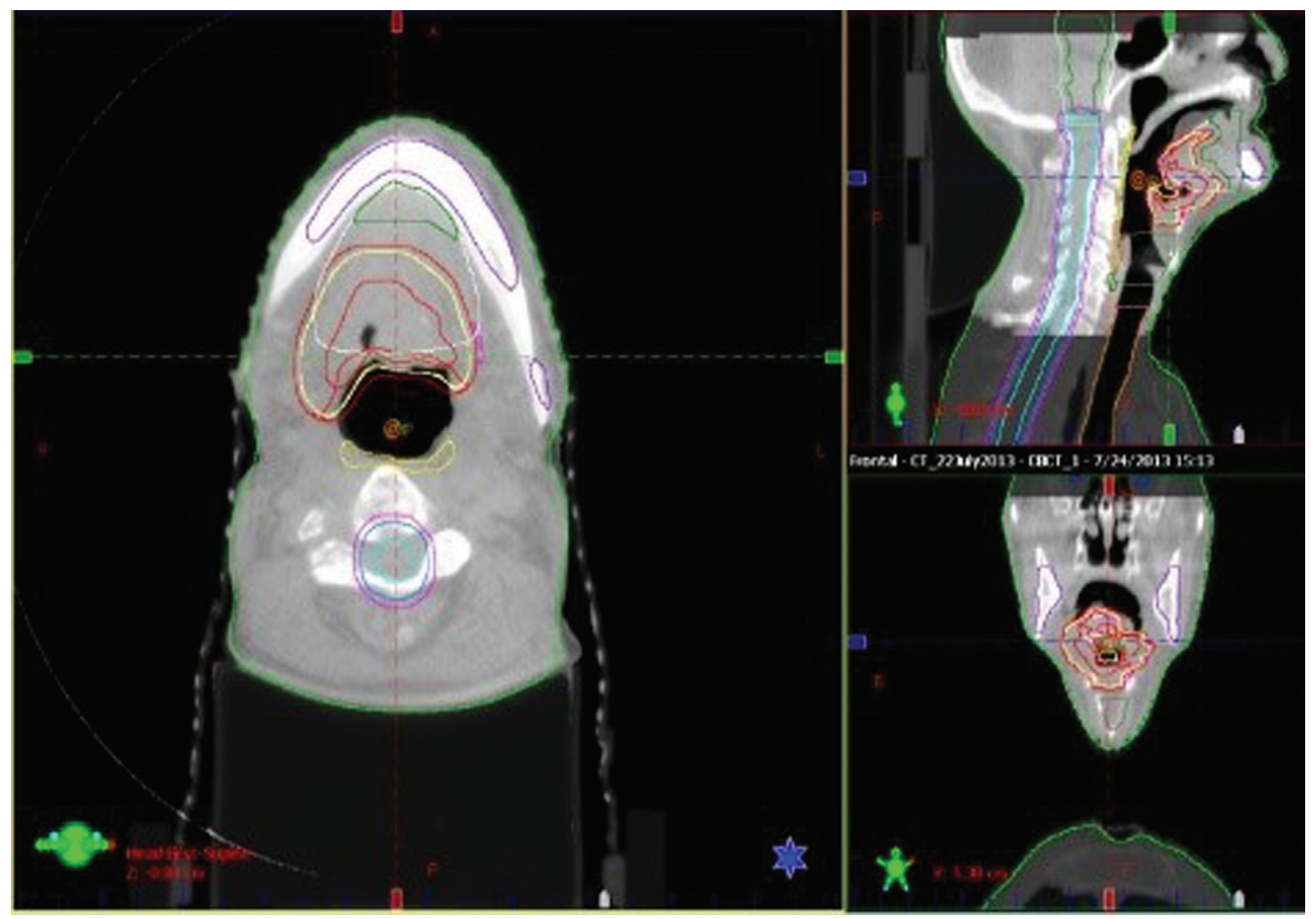

Setup verification by cone beam computed tomography.

\section{Figure 3}

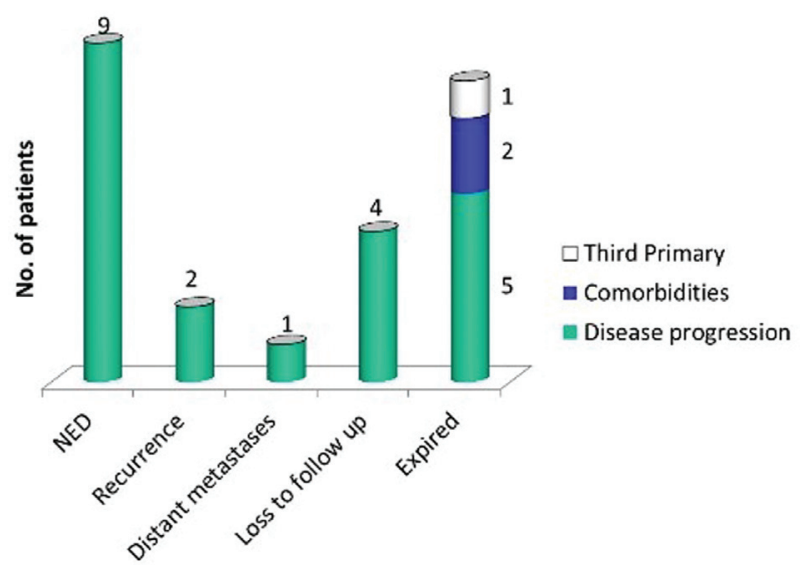

Outcome of patients after reirradiation.

distant metastases, and four (16.6\%) were lost to follow-up (Fig. 3).

\section{Median survival was $\mathbf{3} 3$ months}

Late radiation-induced complications were seen in seven patients, neck fibrosis was seen in four patients, and feeding tube dependency in three patients (Fig. 4).

\section{Discussion}

Following irradiation of advanced HNC, the most common patterns of failure are either LR or the development of a second malignancy [6-11]. Both are difficult to manage when they occur in a previously irradiated area. Surgical resection with or without adjuvant radiotherapy offers the best probability for successful salvage. Unfortunately many patients present at unresectable stage. An increasing interest has developed in the use of concurrent chemotherapy and radiation therapy for formerly irradiated patients with recurrent or second primary HNC. Durable response and survival has been reproducibly demonstrated in a small (15-20\%), but finite minority of patients. Retreatment with conventional techniques to the head-and-neck region can be challenging because of the disease spatially approximating formerly treated dose-limiting anatomy [12]. Because of risk associated with reirradiation, identification of patients who might derive benefit is of utmost importance. The single most important factor is the interval of time from initial radiotherapy treatment. The longer the interval, the less likely the chances of development 


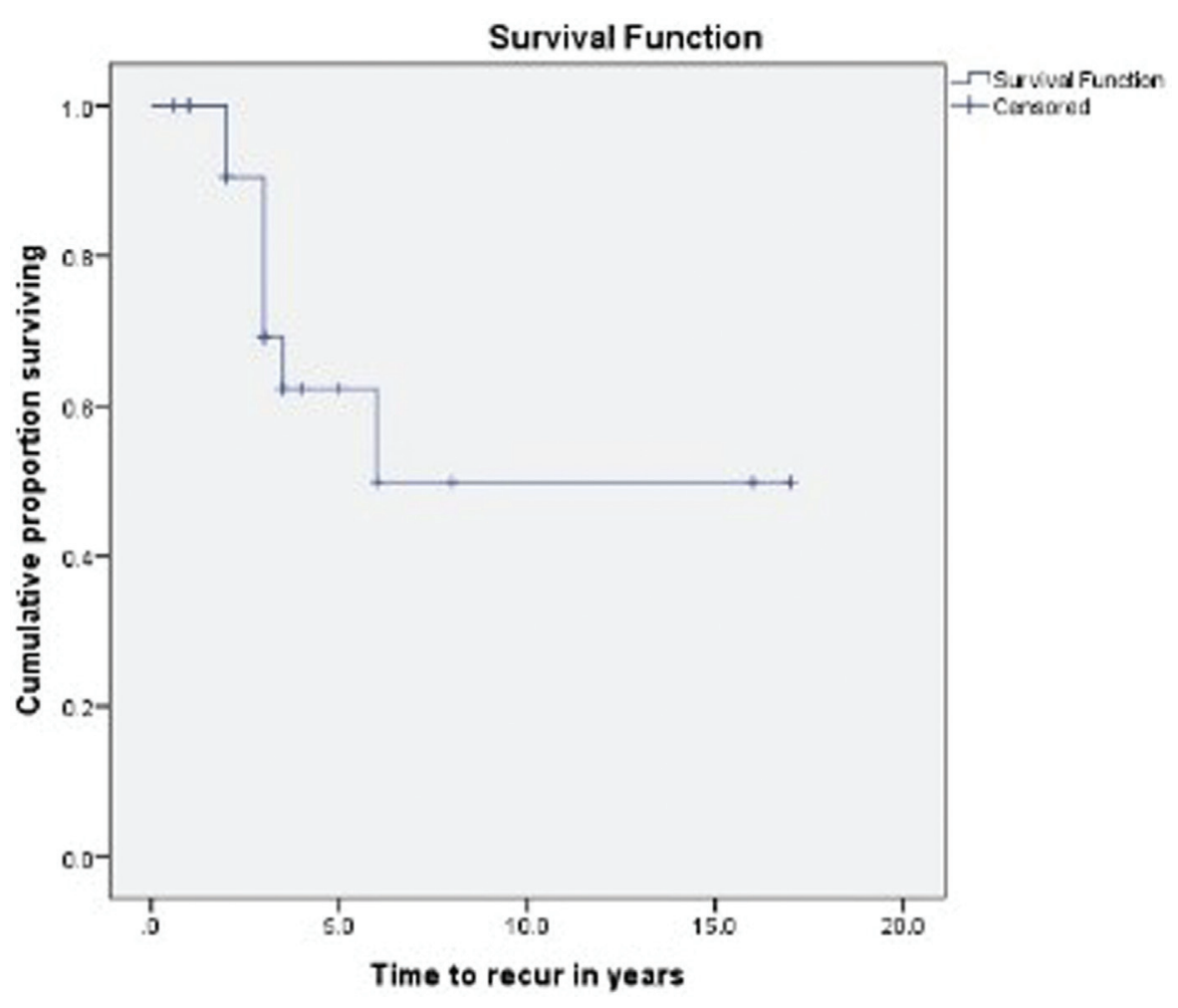

Kaplan-Meier survival curve showing survival pattern of study patients.

of side effects and greater the likelihood of LR control. Also the dose to the critical structures is of importance during reirradiation. Biopsy confirmed evidence of recurrence is imperative. The dose and fractionation schedule used in the setting of reirradiation have varied across the published reports. Higher the dose delivered, greater the probability of local control. Salama et al. [13] reported a 3-year overall survival and LR control of 30 and 56\%, respectively, for patients who received greater than 58 Gy compared with only 6 and 33\% among those who received less than $58 \mathrm{~Gy}$. The role of concurrent chemotherapy remains uncertain with reirradiation to the head and neck $[14,15]$. How and whether chemotherapy affects normal tissue tolerance, in the setting of reirradiation is largely unknown. Previous institutional series have established the dosimetric advantages and practicability of IMRT $[16,17]$. Although IMRT has been universally heralded for its ability to deliver elegantly conformal treatment plans to complex target volumes, this technology is critically based on an inherent assumption that the region of interest lies in the exact same position each day as at the time of simulation [18]. The advantage of IGRT is that it offers a quality threedimensional view of the region of interest with excellent soft-tissue contrast at the time of treatment, thereby allowing patient realignment [19]. In our series of patients, the response and tolerance to treatment was mainly based on the patients' performance status.
The prognostic factors associated with improved outcome following reirradiation include irradiation of new primary cancers versus recurrent tumor, the time interval since previous irradiation, higher radiation dose at the time of reirradiation, and the use of concurrent chemotherapy. Reirradiation of new primary cancers is expected to be better than reirradiation of recurrent tumor, due to the inherent aggressiveness and radio resistance of recurrent lesions. In addition, one would expect that patients reirradiated after a long time from initial irradiation would have improved tumor control rates compared with patients irradiated after shorter intervals, because these tumors grow back quickly and it is less likely that the tumor control would be achieved with reirradiation. It is also instinctive that higher radiation doses are associated with better tumor control, as a dose-response relationship exists for the treatment of primary tumors. Based on the available literature and our own experience, the following recommendations can be made for patients with recurrent disease or a second primary tumor after previous irradiation: If the tumor is resectable, salvage surgery is the mainstay of treatment. Postoperative reirradiation should be considered in patients at high risk of subsequent recurrence (e.g. in cases of positive resection margins and/or extranodal spread). If the disease is unresectable, definitive reirradiation with concurrent chemotherapy can be offered to the patient. Even 
though the likelihood of a positive outcome is limited in these cases, it should be realized that no other curative treatment options are available. Treatment within the context of clinical trials remains warranted to establish evidence-based treatment policies. LR recurrences after radiotherapy in $\mathrm{HNCs}$ represent a challenging clinical problem. Surgical resection, with or without reirradiation, provides the highest likelihood for successful salvage. Toxicity during reirradiation is an important issue. Patients reirradiated after a long time from initial irradiation have better tolerance compared with patients irradiated after shorter intervals. Novel treatment strategies to improve the outcome and minimize late complications in patients with $\mathrm{HNC}$ requiring reirradiation are warranted.

\section{Financial support and sponsorship \\ Nil.}

\section{Conflicts of interest}

There are no conflicts of interest.

\section{References}

1 Lefebvre JL, Rolland F, Tesselaar M, Bardet E, Leemans CR, Geoffrois L, et al. Phase 3 randomized trial on larynx preservation comparing sequential vs alternating chemotherapy and radiotherapy. J Natl Cancer Inst 2009; 101:142-152.

2 Schwartz LH, Ozsahin M, Zhang GN, Touboul E, De Vataire F, Andolenko $\mathrm{P}$, et al. Synchronous and metachronous head and neck carcinomas. Cancer 1994; 74:1933-1938.

3 Goodwin JrWJ. Salvage surgery for patients with recurrent squamous cell carcinoma of the upper aero digestive tract: when do the ends justify the means? Laryngoscope 2000; 110:1-18.

4 Browman GP, Cronin L. Standard chemotherapy in squamous cell head and neck cancer: what we have learned from randomized trials. Semin Oncol 1994; 21:311-319.
5 Duprez F, Madani I, Bonte K, Boterberg T, Vakaet L, Derie C, et al. Intensity-modulated radiotherapy for recurrent and second primary head and neck cancer in previously irradiated territory. Radiother Oncol 2009; 93:563-569.

6 Warren S, Gates O. Multiple primary malignant tumors: a survey of the literature and statistical study. Am J Cancer 1932; 16:1358-1414.

7 Moertel CG, Dockerty MB, Baggenstoss AH. Multiple primary malignant neoplasms. II. Tumors of different tissues or organs. Cancer 1961; 14:231-237.

8 Curtis RE, Freedman DM, Ron E, Ries LA, Hacker DG, Edwards BK, et al. New Malignancies Among Cancer Survivors: SEER Cancer Registries, 1973 -2000. Bethesda, MD: National Cancer Institute 2006: 9-14.

9 Cooper JS, Pajak TF, Forastiere AA, Jacobs J, Campbell BH, Saxman SB, et al. Postoperative concurrent radiotherapy and chemotherapy for highrisk squamous- cell carcinoma of the head and neck. N Engl J Med 2004; 350:1937-1944.

10 Bernier J, Domenge C, Ozsahin M, Matuszewska K, Lefèbvre JL, Greiner $\mathrm{RH}$, et al. Postoperative irradiation with or without concomitant chemotherapy for locally advanced head and neck cancer. $\mathrm{N}$ Engl $\mathrm{J}$ Med 2004; 350:1945-1952.

11 Forastiere AA, Goepfert H, Maor M, Pajak TF, Weber R, Morrison W, et al. Concurrent chemotherapy and radiotherapy for organ preservation in advanced laryngeal cancer. N Engl J Med 2003; 349:2091-2098.

12 Lee N, Chan K, Bekelman JE, Zhung J, Mechalakos J, Narayana A, et al. Salvage re-irradiation for recurrent head and neck cancer. Int $J$ Radiat Oncol Biol Phys 2007; 68:731-740.

13 Salama JK, Vokes EE, Chmura SJ, Milano MT, Kao J, Stenson KM, et al. Long-term outcome of concurrent chemotherapy and reirradiation for recurrent and second primary head-and-neck squamous cell carcinoma. Int J Radiat Oncol Biol Phys 2006; 64:382-391.

14 Biagioli MC, Harvey M, Roman E, Raez LE, Wolfson AH, Mutyala S, et al. Intensity-modulated radiotherapy with concurrent chemotherapy for previously irradiated, recurrence head and neck cancer. Int J Radiat Oncol Biol Phys 2007; 69:1067-1073.

15 Wong SJ, Machtay M, Li Y. Locally recurrent, previously irradiated head and neck cancer: concurrent re-irradiation and chemotherapy,or chemotherapy alone? J Clin Oncol 2006; 24:2653-2658.

16 Sulman EP, Schwartz DL, Le TT, et al. IMRT reirradiation of head and neck cancer - disease control and morbidity outcomes. Int J Radiat Oncol Biol Phys 2009; 73:399-409.

17 Emami B, Bignardi M, Spector GJ, et al. Reirradiation of recurrent head and neck cancers. Laryngoscope 1987; 97:85-88.

18 Popovtzer A, Gluck I, Chepeha DB, et al. The pattern of failure after reirradiation of recurrence squamous cell head and neck cancer: implications for defining the targets. Int J Radiat Oncol Biol Phys 2009; 74:1342-1347.

19 Mackie TR, Kapatoes J, Ruchala K, et al. Image guidance for precise conformal radiotherapy. Int J Radiat Oncol Biol Phys 2003; 56:89-105. 